BUTP-94/7

Imperial/TP/93-94/29

hep-th/9404180

\title{
On string cosmology and the RG flow in $2 d$ field theory
}

\author{
C. Schmidhuber \\ Institute for Theoretical Physics \\ University of Bern, CH-3012 Bern, Switzerland \\ and \\ A.A. Tseytlin间 \\ Theoretical Physics Group, Blackett Laboratory \\ Imperial College, London SWY 2BZ, U.K.
}

\begin{abstract}
Time-dependent solutions of bosonic string theory resemble renormalisation group trajectories in the space of $2 d$ field theories: they often interpolate between repulsive and attractive static solutions. It is shown that the attractive static solutions are those whose spatial sections are minima of $|\bar{c}-25|$, where $\bar{c}$ is the 'c-function'. The size of the domain of attraction of such a solution may be a measure of the probability of the corresponding string vacuum. Our discussion has also an implication for the RG flow in theories coupled to dynamical $2 d$ gravity: the flow from models with $c>25$ to models with $c<25$ is forbidden.
\end{abstract}

$4 / 94$

*e-mail: christof@butp.unibe.ch

†e-mail: tseytlin@ic.ac.uk. On leave from Lebedev Physics Institute, Moscow, Russia 


\section{Introduction}

If the universe is described by string theory, the central charges of the flat spatial coordinates and of the compactification space must add up to $c_{t o t}=25$ ( $\hat{c}_{t o t}=9$ in superstring theory) at the present stage of evolution. Otherwise we would observe a time-dependent dilaton background [1, 2], which - from the world-sheet point of view - is needed to balance the central charge and which would manifest itself in time dependence of coupling constants.

However, one may speculate that $c_{\text {tot }}$ has been larger or smaller than 25 in the early universe. In the present paper we derive a result that supports this proposal. Namely, the space of general time-dependent solutions of classical bosonic string theory contains attractors: they are static solutions with 'spatial' CFT parts which are minima of $|\bar{c}-25|$, where $\bar{c}$ is a version of Zamolodchikov's $c$-function [3] equal to the central charge $\bar{c}=c_{t o t}$ at its extrema. One reason why this is potentially interesting is that it seems to suggest a natural way to assign different probabilities to different compactifications - according to the sizes of the corresponding domains of attraction. Though there is no energy-type criterion for comparing different compact internal spaces with the same central charge, by considering their possible time evolution in the early universe one can thus hope to be able to determine which of them are 'most probable' ones.

To simplify the problem, we shall assume that the evolution of the three 'large' spatial dimensions is decoupled and can be ignored. What we call attractors are thus static attractors for the compact internal space. I In contrast to most previous discussions of the cosmological evolution of the internal space (see e.g. 顿), in which only the moduli (e.g., radii of toroidal compactification) change in time, we are interested in solutions in which the central charge of the internal conformal field theory (CFT) changes. We shall discuss bosonic string theory and ignore the tachyon as usual since it is absent in the superstring generalisation. We shall consider only the leading order in the string coupling expansion, i.e., classical string theory, ignoring possible (non-)perturbative corrections like a dilaton potential.

To derive the above result we shall follow an analogy with the standard renormalisation group flow. As discussed in sections 2 and 3, a generic time-dependent classical solution of the string field equations has much in common with a generic RG trajectory in the space of $2 d$ field theories. Classical solutions of string theory can also be viewed as trajectories in the $2 d$ theory space. For example, a solution of $N+1$ dimensional bosonic string low-energy effective equations is given by the target space fields $(\mu, \nu=0,1, \ldots, N+1)$

$$
G_{\mu \nu}(\vec{x}, t), B_{\mu \nu}(\vec{x}, t), \phi(\vec{x}, t)
$$

where $x^{i}(i=1, \ldots, N)$ are the spatial coordinates, $t$ is time, $G_{\mu \nu}$ is the target space metric, $B_{\mu \nu}$ is the antisymmetric tensor field, and $\phi$ is the dilaton. At least locally, diffeomorphism symmetry and the gauge symmetry associated with the antisymmetric tensor field can be

\footnotetext{
${ }^{1}$ Such a suggestion was made in [2].

${ }^{2}$ The evolution of the three "large dimensions" could in principle be included in the discussion. There may also be non-static attractor solutions; they are not considered here.

${ }^{3}$ For simplicity, we shall ignore all other possible couplings. Our argument can be repeated in more general terms, using perturbative string field theory [0] or Wilson's RG approach [9].
} 
used to set

$$
B_{0 i}=0, G_{00}= \pm 1, G_{0 i}=0 .
$$

Then an $N+1$ dimensional string solution can be represented as a trajectory $\vec{\lambda}(t)$ (with time being the parameter along the trajectory) in the space of $N$-dimensional fields

$$
\vec{\lambda}=\left\{G_{i j}(\vec{x}), B_{i j}(\vec{x}), \phi(\vec{x})\right\}
$$

which is the space of coupling constants of $2 d$ bosonic sigma models with a compact $N-$ dimensional euclidean target space and a curved background world sheet metric [6, 7, 8]

$$
I=\frac{1}{4 \pi \alpha^{\prime}} \int d^{2} \xi\left[\sqrt{g} G_{i j}(\vec{x}) \partial_{\alpha} x^{i} \partial^{\alpha} x^{j}+i \epsilon^{\alpha \beta} B_{i j}(\vec{x}) \partial_{\alpha} x^{i} \partial_{\beta} x^{j}+\alpha^{\prime} \sqrt{g} R^{(2)} \phi(\vec{x})\right] .
$$

The orbits $\vec{\lambda}(t)$ will be compared with the standard RG trajectories in the sigma model (1.2). The RG trajectories interpolate between stable and unstable fixed points in theory space. A generic RG trajectory is attracted to some stable fixed point. As will be explained, similar statements hold for the string solutions $\vec{\lambda}(t)$ because of the 'friction' provided by the time-dependent dilaton (see also [10, 11, 12 [1]).

The similarity between the $\mathrm{RG}$ flow (satisfying the standard first-order RG equations) and time-dependent string solutions (satisfying second-order equations) that we shall exploit here has another interesting interpretation: a string solution $\vec{\lambda}(t)$ represents an RG flow in the presence of $2 d$ gravity [13]. . We shall find an interesting qualitative effect of coupling a $2 d$ field theory to $2 d$ gravity: it makes it impossible to flow from fixed points with $c>25$ to fixed points with $c<25$.

This paper is organised as follows. In section 2 the string equations of motion are represented as equations for $\vec{\lambda}(t)$. They take a simple form if expressed in terms of the $\beta$-functions of the spatial $2 d$ field theory. Although obtained to lowest order in $\alpha^{\prime}$, these equations should be exact in $\alpha^{\prime}$ at the vicinity of static solutions. In section 3 the equations are compared with the RG flow equations and the result stated above is derived. In section 4 we consider implications for the RG flow in the presence of $2 d$ gravity. As an illustration of the preceding discussion, the example of the group space sigma model with a Wess-Zumino term is considered in section 5. The corresponding solutions describe an interpolation between flat and compactified space.

Section 6 contains some concluding remarks.

\footnotetext{
${ }^{4}$ For a different discussion of a 'friction' in string theory, an interpolation between fixed points, and the RG flow, see 14 .

${ }^{5}$ For discussions of a relation between the RG flow with dynamical $2 d$ gravity and string theory see, e.g., [15, 16, 17, 10, 5, 18] and also [19, 20, 21, 22, 14.

${ }^{6}$ It makes sense to discuss CFT's with $c>25$ coupled to gravity in the approach of [23, 24], e.g., as bosonic sectors of superconformal field theories coupled to supergravity. One should not worry about the 'wrong' sign of the kinetic term of the Liouville factor $t$ for $\bar{c}>25$ : the associated ghosts should decouple according to the no-ghost theorem for critical string theory $\left(\bar{c}+c_{t}=26\right)$.
} 


\section{Equations of motion for the couplings $\vec{\lambda}(t)$}

If a string solution $\vec{\lambda}(t)$ asymptotically approaches a static solution ('fixed point'), $\vec{\lambda}(t)$ obeys a simple equation of motion at the vicinity of this fixed point, which will be derived below.

\subsection{String effective field equations and $\beta$-functions}

Consider the world-sheet theory (1.2). The corresponding leading-order $\beta$-functions (Weyl anomaly coefficients) for $G_{i j}, B_{i j}$ and $\phi$ can be represented in the form [8, []

$$
\begin{aligned}
\beta_{i j}^{G(N)} & =\alpha^{\prime}\left(R_{i j}+2 \nabla_{i} \nabla_{j} \phi-\frac{1}{4} H_{i m n} H_{j}^{m n}\right), \\
\beta_{i j}^{B(N)} & =\alpha^{\prime}\left(-\frac{1}{2} \nabla_{m} H_{i j}^{m}+H_{i j}^{m} \partial_{m} \phi\right), \\
\tilde{\beta}^{\phi(N)} & =\beta^{\phi(N)}-\frac{1}{4} G^{i j} \beta_{i j}^{G(N)}=\frac{1}{6}\left[C^{(N)}(\vec{x})-26\right] .
\end{aligned}
$$

Here $H_{i j k}=3 \nabla_{[i} B_{j k]}$ and the function

$$
C^{(N)}(\vec{x})=N-\frac{3}{2} \alpha^{\prime}\left[R-\frac{1}{12} H^{2}-4(\nabla \phi)^{2}+4 \square \phi\right]
$$

becomes $\vec{x}$-independent and equal to the central charge when the sigma model represents a conformal theory [25]. Eqs.(2.1)-(2.3) can be derived from the $N$-dimensional target space action [7, 8] (for a review see [26])

$$
S^{(N)}=\int d^{N} x \sqrt{G} e^{-2 \phi}\left[C^{(N)}(\vec{x})-26\right] .
$$

The string effective equations for $\vec{\lambda}(t)$ are the requirements that the $\beta$-functions of the sigma model with $N+1$ dimensional target space (and the additional couplings in (1.1)) vanish. Writing (2.1)-(2.3) in $N+1$ dimensions and making the $N+1$ split we find the following expressions in terms of the $\beta$-functions of the sigma model with $N$-dimensional target space (in what follows we set $\alpha^{\prime}=2$ ):

$$
\begin{aligned}
0=\beta_{00}^{G(N+1)} & =2 \ddot{\varphi}-\frac{1}{2} G^{i k} G^{j l}\left(\dot{G}_{i j} \dot{G}_{k l}+\dot{B}_{i j} \dot{B}_{k l}\right), \\
0=\beta_{i j}^{G(N+1)} & =\beta_{i j}^{G(N)}-G^{00}\left[\ddot{G}_{i j}-\dot{\varphi} \dot{G}_{i j}-G^{m n}\left(\dot{G}_{i m} \dot{G}_{j n}-\dot{B}_{i m} \dot{B}_{j n}\right)\right], \\
0=\beta_{i j}^{B(N+1)} & =\beta_{i j}^{B(N)}-G^{00}\left(\ddot{B}_{i j}-\dot{\varphi} \dot{B}_{i j}-2 G^{k l} \dot{G}_{k[i} \dot{B}_{j] l}\right), \\
0=C^{(N+1)}-26 & =C^{(N)}-25-3 G^{00}\left(\ddot{\varphi}-\dot{\varphi}^{2}\right),
\end{aligned}
$$

where we introduced the shifted dilaton

$$
\varphi=2 \phi-\log \sqrt{G}
$$

The dilaton equation (2.9) can also be written in terms of the $\beta$-function $\beta^{\phi(N)}$ in (2.3) to keep the analogy with (2.7),(2.8). The above equations follow from the action!

$$
S^{(N+1)}=\int d t d^{N} x \sqrt{\left|G^{00}\right|} e^{-\varphi}\left\{C^{(N)}(\vec{x})-25+3 G^{00}\left[\dot{\varphi}^{2}-\frac{1}{4} G^{i k} G^{j l}\left(\dot{G}_{i j} \dot{G}_{k l}+\dot{B}_{i j} \dot{B}_{k l}\right)\right]\right\} .
$$

\footnotetext{
${ }^{7}$ In the simplest case of homogeneous $x^{i}$-independent fields the resulting system of equations is the same as the $O(N, N)$ duality invariant system in [27].
} 
A combination of (2.6) and (2.9) is a constraint. The additional two constraints

$$
\begin{gathered}
0=\beta_{0 i}^{G(N+1)}=\nabla_{k}\left(G^{k l} \dot{G}_{l i}\right)-\frac{1}{2} \dot{B}_{k l} H_{i}{ }^{k l}+2 \partial_{i} \dot{\varphi}-G^{k l} \dot{G}_{l i} \partial_{k} \varphi, \\
0=\beta_{0 i}^{B(N+1)}=-G_{i k} \partial_{j}\left(G^{k l} G^{j n} \dot{B}_{n l}\right)+2 \dot{B}_{i j} \nabla^{j} \varphi
\end{gathered}
$$

further restrict the initial values of the fields and their time derivatives. We shall assume that these constraints can be ignored in the context of our discussion.

It is useful for the following to split the shifted dilaton $\varphi(\vec{x}, t)$ into an $\vec{x}$-dependent part $\tilde{\varphi}(\vec{x}, t)$ and an $\vec{x}$-independent part $\varphi_{0}(t)$ as follows:

$$
\varphi_{0}(t) \equiv-\log \left[\int d^{N} x e^{-\varphi(\vec{x}, t)}\right], \quad \tilde{\varphi}(\vec{x}, t) \equiv \varphi(\vec{x}, t)-\varphi_{0}(t) .
$$

$\varphi_{0}$ is thus minus the logarithm of the proper space volume (at fixed $t$ )

$$
V^{(N)} \equiv \int d^{N} x \sqrt{G} e^{-2 \phi}=e^{-\varphi_{0}}
$$

Let us define the space average of a function $f(\vec{x})$ by

$$
<f(\vec{x})>\equiv \frac{\int d^{N} x f(\vec{x}) e^{-\varphi(\vec{x})}}{\int d^{N} x e^{-\varphi(\vec{x})}} .
$$

An important role is played by the function

$$
Q(t) \equiv-\dot{\varphi}_{0}(t)=-<\dot{\varphi}(\vec{x}, t)>
$$

As follows from (2.6), at fixed points $\dot{G}_{i j}=\dot{B}_{i j}=0, \ddot{\varphi}=\ddot{\phi}=0$ so that $Q$ is constant and $\varphi_{0}(t)=-\frac{1}{2} Q t+$ const, but in general $Q$ will depend on $t$. Integrating (2.9) weighted by $e^{-\varphi}$ yields

$$
\dot{Q}+Q^{2}=-\frac{1}{3} G^{00}(\bar{c}-25)
$$

where (2.12) has been used and we have defined the function

$$
\bar{c}(t)=\bar{c}(G, B, \varphi)=<C^{(N)}(\vec{x})>=\frac{S^{(N)}}{V^{(N)}}+26 .
$$

$S^{(N)}$ is the action in (2.5). $\bar{c}$ (or, more precisely, $S^{(N)}$ ) can be considered a generalisation of the 'c-function' [3] to the case of sigma models with dilaton coupling [28, 29].9] Eq. (2.6) implies that

$$
\begin{aligned}
\dot{Q} & =<-\ddot{\varphi}+\dot{\varphi}^{2}-Q^{2}>=<(\dot{\varphi}-<\dot{\varphi}>)^{2}-\ddot{\varphi}> \\
& =<\dot{\tilde{\varphi}}^{2}-\frac{1}{4} G^{i k} G^{j l}\left(\dot{G}_{i j} \dot{G}_{k l}+\dot{B}_{i j} \dot{B}_{k l}\right)>.
\end{aligned}
$$

${ }^{8}$ This is clear if the constraints on fields and their first time derivatives can be solved for arbitrary $G_{i j}, B_{i j}$, $\phi$, which is likely to be the case in perturbation theory in $t$ near the fixed points that we shall mostly consider.

${ }^{9}$ Note that if $\varphi$ is used as the independent dilaton coupling then the equations for $G_{i j}$ and $B_{i j}$ that follow from the variation of $\bar{c}$ and $S^{(N)}$ are equivalent. 


\subsection{Equations at the vicinity of fixed points}

In the following we shall study the behavior of solutions which asymptotically approach fixed points. By a fixed point of the above equations we mean a solution in which all time derivatives are zero, except for $\dot{\varphi}_{0}$ or (what is the same at a fixed point) $\dot{\phi}_{0}$, where $\phi_{0}$ is the spatially constant mode of the dilaton. We thus allow for a possible $\vec{x}$-independent dilaton background that grows linearly in time (the sigma model with $N$-dimensional target space obviously does not 'feel' an $\vec{x}$-independent dilaton background).

First let us consider the case when $c \neq 25$. Within an appropriate neighbourhood of the fixed point, both $\bar{c}(G, B, \phi)$ and $C^{(N)}(x)$ can be approximated by a constant $c$. As follows from (2.6), (2.17), to lowest order $\ddot{\varphi}=0, \ddot{\tilde{\varphi}}=0, \dot{Q}=0$. Eq.(2.14) then reduces to

$$
Q^{2}=-\frac{1}{3} G^{00}(c-25)
$$

In order to have a real dilaton, the target space must have euclidean signature $\left(G^{00}=+1\right)$ at the vicinity of fixed points with $c<25$, and minkowskian signature $\left(G^{00}=-1\right)$ at the vicinity of fixed points with $c>25$. Ignoring small terms which are quadratic in first derivatives of the couplings, eqs.(2.7)-(2.9) can be represented in the form

$$
\begin{aligned}
\ddot{\vec{\lambda}}+Q \dot{\vec{\lambda}} & =\left\{\begin{array}{r}
-\vec{\beta} \text { for } c>25 \\
+\vec{\beta} \text { for } c<25,
\end{array} \text { with } Q^{2}=\frac{1}{3}|c-25|,\right. \\
\text { where } \vec{\lambda} & \equiv\left\{G_{i j}(x), B_{i j}(x)\right\} .
\end{aligned}
$$

Here, $\vec{\beta}$ are the $\beta$-functions (Weyl anomaly coefficients) of the sigma model with $N$-dimensional target space.

Next, consider a vicinity of a fixed point with $c=25$. As follows from (2.14), $Q(t)$ can no longer be approximated by a non-zero constant. Let us determine $Q(t)$ to the next order in couplings. Let us first argue that $\dot{\varphi}(t)$ can still be approximated by $-Q(t)$ : According to (2.3), 2.4) the $\beta$-function for the $\vec{x}$-dependent modes $\tilde{\varphi}(x)$ of $\varphi(x)$ has a linear piece with plane waves $\tilde{\varphi}_{p}\left(\sim a_{p} \cos p x\right)$ as eigenvectors. From eq.(2.9), $a_{p}(t)$ has some exponential $t$ dependence. Eq.(2.6), expanded in Fourier modes, relates the amplitudes $\tilde{\varphi}_{p}$ to the amplitudes

of perturbations of $B$ and $G$. If the latter are of order $\epsilon, \tilde{\varphi}_{p}$ is of order $\epsilon^{2}$ so that $\dot{\tilde{\varphi}}^{2}$ is of order $\epsilon^{4}$ and can be neglected in (2.17). Eq. (2.17) then takes the form

$$
\dot{Q}=-\frac{1}{4} \dot{\vec{\lambda}}^{2}
$$

where $\dot{\vec{\lambda}}^{2}$ is defined by averaging as in (2.12)

$$
\dot{\vec{\lambda}}^{2} \equiv<G^{i k} G^{j l}\left(\dot{G}_{i j} \dot{G}_{k l}+\dot{B}_{i j} \dot{B}_{k l}\right)>.
$$

Combining (2.20) with eq. 2.14) yields:

$$
Q^{2}=-\frac{1}{3} G^{00}[\bar{c}(\vec{\lambda})-25]+\frac{1}{4} \dot{\vec{\lambda}}^{2},
$$


where the constant $c$ is now replaced by the $c$-function $\bar{c}$. Whether $G^{00}$ should be chosen positive or negative now depends on the behavior of $\bar{c}(\vec{\lambda})$ at the vicinity of the fixed point. Eq.(2.22) implies that if $\dot{\lambda}$ is of order $\epsilon$ then $Q$ is also of order $\epsilon$. Thus,

$$
|Q|=\left|\dot{\varphi}_{0}\right| \sim O(\epsilon) \gg|\dot{\tilde{\varphi}}| \sim O\left(\epsilon^{2}\right)
$$

and so $\dot{\varphi}$ in eqs.(2.7),(2.8) can be approximated by $-Q(t) . \square$ This yields:

$$
\ddot{\vec{\lambda}}+Q \dot{\vec{\lambda}}+O\left(\dot{\lambda}^{2}\right)= \begin{cases}-\vec{\beta} & \text { for } G^{00}=-1 \\ +\vec{\beta} & \text { for } G^{00}=+1\end{cases}
$$

The $O\left(\dot{\lambda}^{2}\right)$ terms are the $\dot{G}^{2}, \dot{B}^{2}, \dot{G} \dot{B}$ terms in (2.7)-(2.8). Note that they can be removed by redefining the variables: for example, the $\dot{G}^{2}$ terms are removed by changing variables to $\lambda_{j}^{i}$ such that $\dot{\lambda}_{j}^{i}=G^{i k} \dot{G}_{k j}$ (in the basis where the metric is diagonal, $G_{k j}=\delta_{k j} g_{j}$, $\left.\lambda_{j}^{i}=\delta_{j}^{i} \log g_{j}\right)$.

The equations (2.23), (2.18) have been derived to leading order in $\alpha^{\prime}$. However, at the vicinity of fixed points where time derivatives of $\vec{\lambda}$ are small, they are actually good approximations, with $\vec{\beta}$ containing all orders in $\alpha^{\prime}$ (see also sec. 4 ).

\section{First application: attractive and repulsive fixed points}

Depending on their initial parameters, classical solutions of string theory are either attracted to fixed points or they diverge as $t \rightarrow \infty$. By attractive fixed points we understand static solutions that attract non-static ones within some finite volume domain of initial parameters (by a finite - as opposed to infinitesimal - volume domain we mean that no initial parameters are fine-tuned). Let us now use the equations of the last section to argue that attractive fixed points exist and correspond to minima of $|\bar{c}(\vec{\lambda})-25|$, where the $c$-function $\bar{c}$ was defined in (2.15). We begin with fixed points with $c \neq 25$. As shown, if a solution asymptotically approaches a fixed point with $c \neq 25$, near the fixed point it obeys eq.(2.18), i.e.,

$$
\ddot{\vec{\lambda}}+Q \dot{\vec{\lambda}}=\left\{\begin{array}{ll}
-\vec{\beta} & \text { for } c>25, \\
+\vec{\beta} & \text { for } c<25,
\end{array} \text { with } Q^{2} \approx \frac{1}{3}|c-25|=\right.\text { const }
$$

To identify the attractors, let us compare (3.1) with the standard renormalisation group flow equation for the $N$-dimensional theory,

$$
\dot{\vec{\lambda}}= \begin{cases}-\vec{\beta} & \text { towards the IR, } \\ +\vec{\beta} & \text { towards the UV. }\end{cases}
$$

The time in (3.1) is different from RG 'time' in (3.2), but the $\vec{\beta}$ functions are the same."1 This similar structure of eqs.(3.1), (3.2) leads to the following simple remarks (see also [10, 11, 12] for some of the points; note also the discussion in ref. [14]):[7]

\footnotetext{
${ }^{10}$ The fact that $Q \geq \epsilon$ while $\dot{Q}$ is of order $\epsilon^{2}$ signals the behavior $Q \sim t^{-1}$ (see also the example in sec. 5).

${ }^{11}$ The $\beta$-functions in eq.(3.2) are assumed to be defined with specific 'diffeomorphism terms' so that they represent the Weyl anomaly coefficients [30, 31].

${ }^{12}$ It should be kept in mind that since the order of these two equations is different, there are different sets of initial conditions. Also, recall that we are ignoring the gauge constraints present in the string system of equations.
} 
1. Fixed points: Both equations describe a motion of a particle in $\vec{\lambda}$-space with the $\beta$-function as a driving force. They obviously have the same fixed points, namely conformal field theories $(\vec{\beta}=0)$.

2. Sign of $Q$ : If $Q$ is chosen to be positive, the motion (3.1) is damped. If $Q$ is chosen to be negative, it is anti-damped. An anti-damped motion is the time-reversal of a damped motion. It has no stable fixed points - any small perturbation near a fixed point will blow up (sometimes preceded by growing oscillations). Reaching a fixed point would require fine-tuning the initial parameters. In the following we are interested in stable fixed points of (3.1); for this purpose it is sufficient to consider the sector of solutions with positive $Q$.

3. Stability of fixed points: Since the motion with positive $Q$ is damped, it follows from the sign in front of $\vec{\beta}$ in (3.1), that the stable fixed points of (3.1) with $c>25$ are the IR-stable fixed points of (3.2). This is clear since the only difference between the two equations is that the motion (3.2) is infinitely damped while (3.1) is finitely damped. Thus solutions of (3.1) may oscillate around stable fixed points but will eventually settle down there. On the other hand, the stable fixed points of (3.1) with $c<25$ are the UV-stable fixed points of (3.2). To summarise, the stable fixed points of (3.1) are the minima of $|\bar{c}-25|, \bar{c}$ being the (generalised) $c$-function [3, 28]. In other words, they are conformal field theories that contain no relevant operators for $c>25$ or no irrelevant operators for $c<25$ (see a discussion below).

4. Consequence of the c-theorem [3]: Since (3.1) differs from the equation for RG trajectories (3.2) only by finite vs. infinite damping, it is clear that cosmological solutions of (3.1) also obey an analog of the $c$-theorem: if a solution of (3.1) interpolates between two unitary CFT's, an unstable one with a central charge $c_{1}$ and a stable one with a central charge $c_{2}$ and if both $c_{1}, c_{2}>25$ then $c$ decreases in the sense that $c_{2}<c_{1}$. If $c_{1}, c_{2}<25, c$ increases instead, $c_{2}>c_{1}$.

5. Moduli directions: If a CFT has a modulus, then (3.2) does not allow a flow in this direction. By contrast, (3.1) allows 'rolling' along the moduli directions, which has been exploited in some cosmological string solutions (see e.g. [4, [27]). For such solutions, $\bar{c}$ stays constant in time. In this paper, the focus is instead on cosmological solutions with time-dependent $\bar{c}$. For solutions with $Q>0$, any 'rolling' along moduli directions will come to rest after a while due to dilaton damping, at least to leading order in $\dot{\lambda}$.

6. Limit $|c| \rightarrow \infty$ : For $c \rightarrow \pm \infty, Q \rightarrow \infty$. In the case when $c \rightarrow+\infty$, a solution of (3.1) becomes identical to the flow (3.2) towards the IR region if $2 t / Q$ is identified with ' $\mathrm{RG}$ time'. In the case $c \rightarrow-\infty$, the solution of (3.1) is identical to the RG flow towards the UV region.

Let us now also consider fixed points with $c=25$. Then $Q(t)$ is small and not obviously positive, so the issue of stability is more subtle. Let us show that IR or UV stable fixed points of the RG flow are still stable fixed points of the string equations of motion. According to 
Sect.2.2, in the vicinity of fixed points with $c=25$ :

$$
\begin{aligned}
\ddot{\vec{\lambda}}+Q \dot{\vec{\lambda}}+O\left(\dot{\lambda}^{2}\right) & =G^{00} \vec{\beta} \\
Q^{2}(t) & =-\frac{1}{3} G^{00}[\bar{c}(\vec{\lambda})-25]+\frac{1}{4} \dot{\vec{\lambda}}^{2} \\
\dot{Q}(t) & =-\frac{1}{4} \dot{\vec{\lambda}}^{2}
\end{aligned}
$$

The last equation states that $Q$ decreases, either to $-\infty$ or until a fixed point is reached. Suppose the fixed point with $c=25$ is IR-stable and such that $\bar{c}(\vec{\lambda})-25$ is positive definite, and zero only at the fixed point. To have a real $Q, G^{00}=-1$ must be chosen. At the vicinity of the fixed point, consider a damped motion with $Q>0$. From (3.5), $Q(t)$ will decrease and become 0 at some point. Then (3.4) implies that the fixed point has been reached: $\dot{\vec{\lambda}}=0, c=25$. Eqs. (3.3) and (3.5) imply that the trajectory will stay at the fixed point: $\dot{Q}=0, \ddot{\vec{\lambda}}=0, \ddot{Q}=0, \ldots$, etc. So IR stable fixed points of the RG flow with $c=25$ are also fixed points of the above equations. Conversely, IR unstable saddle points of the flow are also unstable fixed points of the string equations: since $c(\vec{\lambda})-25$ is not positive definite then, there are solutions with $Q=0, \dot{\vec{\lambda}} \neq 0 \rightarrow \dot{Q}<0$, so $Q$ becomes negative and the flow anti-damped. Analogous arguments can be applied for Euclidean signature and UV stable fixed points. For an example of a solution approaching a fixed point with $c=25$, see sec. 5 .

In summary, the attractors of time-dependent string solutions are CFT's with $c \geq 25$ and no relevant operators, or CFT's with $c \leq 25$ and no irrelevant operators. One can always find 'minisuperspace' examples for such theories, as the one in sec. 5. Beyond minisuperspace approximations, CFT's with no irrelevant operators do not exist: in general, perturbations of the form $\cos \vec{p} \vec{x}$ with arbitrarily high $\vec{p}^{2}$ and therefore dimension can be turned on.

On the other hand, CFT's with no relevant operators exist, provided we ignore the nonderivative operators, corresponding to the tachyon. At the linear order in the couplings, 'no relevant operators' means 'no operators with dimension less than two'. Such operators would correspond to tachyons in the low-energy effective string theory and should be absent in all candidates for (super)string vacua. To next order, the quadratic piece of the $\beta$-function of a marginal (dimension 2) operator must also be non-negative. That this is a nontrivial requirement will be seen on the example in sec. 5 .

\section{Second application: RG flow in the presence of $2 d$ gravity}

The similarity of the string equations of motion (3.1) and the RG flow equations (3.2) has an interesting interpretation: special solutions of (3.1) can be interpreted as renormalisation group trajectories in the presence of $2 d$ gravity [15, 10, 18]. Let us briefly review this connection and apply the preceding discussion to conclude that the presence of gravity does not allow to flow from a fixed point with $c>25$ to a fixed point with $c<25$.

\footnotetext{
${ }^{13}$ I.e., we assume the absence of moduli directions for simplicity.

${ }^{14}$ As explained in the introduction, we will not worry about the tachyon for $c>1$ and ghosts for $c \geq 25$.
} 
Consider a CFT with $N$ fields $x^{i}$, central charge $c$ (we choose $c \neq 25$ ) and world-sheet action $I_{\text {cft }}(\vec{x})$, perturbed by interactions $\Phi_{i}(\vec{x})$ with small coupling constants $\lambda^{i}$ and scaling dimensions $h_{i}$ :

$$
I^{(N)}=I_{\mathrm{cft}}(\vec{x})+\lambda^{i} \int d^{2} \xi \Phi_{i}(\vec{x}) .
$$

Without gravity, the $\beta$-functions for $\lambda^{i}$ are (see, e.g, [32])

$$
\beta^{i}=\left(h_{i}-2\right) \lambda^{i}+\pi c_{j k}^{i} \lambda^{j} \lambda^{k}+\ldots
$$

with $c_{j k}^{i}$ being OPE coefficients. The model (4.1) coupled to $2 d$ gravity must be generally covariant, i.e. should be described (in the conformal gauge [23, 24]) by a conformally invariant theory with an additional field $t$ related to the conformal factor of the world-sheet metric [39, 16, 17, 10]. In the context of perturbation theory in $\lambda^{i}$ one finds to quadratic order:町

$$
\begin{aligned}
I=I_{\mathrm{cft}}(\vec{x})+\frac{1}{8 \pi} \int d^{2} \xi \sqrt{\hat{g}}\left[ \pm(\partial t)^{2}-Q R^{(2)} t\right]+\int d^{2} \xi \lambda^{i}(t) \Phi_{i}(\vec{x}), \\
\lambda^{i}(t)=\lambda^{i} e^{\alpha_{i} t}+\frac{\pi}{Q \pm 2 \alpha_{i}} c_{j k}^{i} \lambda^{j} \lambda^{k} t e^{\alpha_{i} t}+O\left(\lambda^{3}\right), \\
Q=\sqrt{\frac{|25-c|}{3}}, \\
\alpha_{i}^{2}+Q \alpha_{i}= \begin{cases}h_{i}-2 & \text { for } c \geq 25 \\
2-h_{i} & \text { for } c \leq 1 .\end{cases}
\end{aligned}
$$

The kinetic term for $t$ in (4.3) has a plus sign for $c \leq 1$ and a minus sign for $c \geq 25$ in order to have real $Q$. The $\mathrm{O}\left(\lambda^{2}\right)$ terms in (4.4), introduced in [19], are needed to insure conformal invariance at quadratic order in $\lambda$ if there are nontrivial OPE coefficients (see also [20, 21, 22]). By construction, $\vec{\lambda}(t)$ is a solution of the conformal invariance equations (i.e. string equations of motion in $N+1$ dimensions) derived using perturbation theory in powers of couplings $\lambda^{i}$ near an $N$-dimensional conformal point. Indeed, it is easily seen from (4.6) that $\vec{\lambda}(t)$ in (4.4) obeys (3.1) up to order $\lambda^{2}$.

It is conjectured that $\lambda^{i}(t)$ in eq.(4.3) can be interpreted as running coupling constants of the theory (4.1) coupled to gravity. More precisely, $t$ can be related to 'renormalization group time' $\tau$ via the cosmological constant operator $\square$ which defines the area of the surface, e.g,

$$
A=\int d^{2} \xi \sqrt{g}=\int d^{2} \xi \sqrt{\hat{g}} e^{\alpha t}=e^{-2 \tau} \Rightarrow t=-\frac{2}{\alpha} \tau, \quad \alpha=-\frac{Q}{2}+ \begin{cases}\frac{1}{2} \sqrt{Q^{2}+8} & \text { for } c \geq 25 \\ \frac{1}{2} \sqrt{Q^{2}-8} & \text { for } c \leq 1 .\end{cases}
$$

\footnotetext{
${ }^{15}$ Here target space gauge invariance has been used to eliminate other terms with derivatives of $t$.

${ }^{16}$ This confirms again that (3.1) is actually exact near fixed points since it is reproduced both in the two complementary approaches - loop (or 'power of $\alpha^{\prime \prime}$ ) expansion and perturbative (or 'power of coupling') expansion.

${ }^{17}$ Such identification was considered also in $[14$.
} 
$\tau$ is defined such that $\tau \rightarrow \infty$ corresponds to the UV. Without coupling to gravity, the coupling constant flow is determined by (4.2). With gravity switched on, one finds from (4.4):

$$
\frac{d}{d \tau} \lambda^{i}=\left(\tilde{h}_{i}-2\right) \lambda^{i}+\pi \tilde{c}_{j k}^{i} \lambda^{j} \lambda^{k}+\ldots
$$

with modified coefficients of the $\beta$-functions

$$
\begin{aligned}
\tilde{h}_{i}-2 & =-\frac{2}{\alpha} \alpha_{i} \\
\tilde{c}_{j k}^{i} & =-\frac{2}{\alpha} \frac{1}{\left(Q+2 \alpha_{i}\right)} c_{j k}^{i} .
\end{aligned}
$$

There is evidence that (4.4) indeed correctly describes the flow in the presence of gravity: (i) the phase diagram of the sine-Gordon model coupled to gravity, derived in this way [19], agrees with the matrix model results [34], and (ii) the quadratic parts [19] of the $\beta$-functions in (4.8) for the case $\alpha_{i}=0$ agree [21] with the light-cone gauge computation [20].

Let us determine which ends of the $t$-line correspond to the infrared and the ultraviolet regions. At fixed points with central charge $c$, (4.7) yields:

$$
\alpha \text { is }\left\{\begin{array} { l l } 
{ > 0 } & { \text { for } c \geq 2 5 , } \\
{ < 0 } & { \text { for } c \leq 1 }
\end{array} \Rightarrow \text { IR corresponds to } \left\{\begin{array}{ll}
t \rightarrow+\infty & \text { for } c \geq 25 \\
t \rightarrow-\infty & \text { for } c \leq 1
\end{array}\right.\right.
$$

For $1<c<25$, the relation between $t$ and $\tau$ is complex (we shall not discuss this case here).

Since we have seen in section 3 that the attractive fixed points of string solutions are at $t \rightarrow+\infty$ ( $t \rightarrow-\infty$ corresponds to anti-damping), one might conclude that there are no IR stable fixed points with $c \leq 1$ in the presence of gravity. This is, however, not the case. The point is that only special solutions of string theory can be interpreted as RG trajectories in the presence of $2 d$ gravity. In the theory of $2 d$ gravity coupled to matter, we are instructed to impose a boundary condition on $\lambda^{i}(t)$ in (4.3): $\alpha_{i}$ in the couplings $\lambda^{i}(t)$ must have the values $\alpha_{i}^{+}$in

$$
\alpha_{i}^{ \pm}= \begin{cases}-\frac{Q}{2} \pm \sqrt{\frac{Q^{2}}{4}-\left(h_{i}-2\right)} & \text { for } c \geq 25 \\ -\frac{Q}{2} \pm \sqrt{\frac{Q^{2}}{4}+\left(h_{i}-2\right)} & \text { for } c \leq 1\end{cases}
$$

i.e. it must not take the 'wrong' Liouville dressing values $\alpha_{i}^{-}$, corresponding to 'non-existing' operators and states of Liouville theory [35, 36, 37]. This boundary condition is required, in particular, for an agreement with matrix model results and with the flow without gravity in the limit $|c| \rightarrow \infty$.

What does it mean to drop the negative Liouville dressings? Consider a fixed point with $c<1$ and an irrelevant direction, $h_{i}>2$; in that case the flow without gravity converges to a fixed point in the IR. Then $\alpha_{i}^{+}>0, \alpha_{i}^{-}<0$, so that $e^{\alpha_{i}^{+} t}$ goes to zero in the $\operatorname{IR}(t \rightarrow-\infty)$,

\footnotetext{
${ }^{18}$ In the matrix model, one can turn on negative powers of matrices in the matrix model potential, which have been conjectured to correspond to the operators with the 'wrong' Liouville dressing (see, e.g., 38]). It would be very interesting to see if these negative powers have an interpretation in terms of random lattices. Then the additional boundary condition could be dropped and there would be no distinction between the flow in the presence of gravity and string theory solutions.
} 
while $e^{\alpha_{i}^{-} t}$ diverges in the IR. For string solutions, both choices are allowed. The statement that the motion is anti-damped and that the fixed point is therefore unstable at $t \rightarrow \infty$ is precisely the statement that the general linear combination of both solutions diverges at $t \rightarrow \infty$. But for the RG flow in the presence of gravity the solutions that contain $\alpha_{i}^{-}$are not allowed. Under this restriction, the flow does have IR stable fixed points with $c \leq 1$ : as without gravity, they are minima of $\bar{c}$.

One may then wonder whether there are any qualitative changes in the RG flow due to coupling to $2 d$ gravity, apart from the quantitative modification of the $\beta$ - function coefficients. Let us conclude by pointing out two qualitative effects. First, being second order in derivatives, the flow in the presence of gravity can oscillate around fixed points before settling down at them (cf. (3.2) and (3.1)). As a consequence, the $\bar{c}$-function does not strictly decrease along RG trajectories. ${ }^{10}$ Using (3.1) and the ordinary $c$-theorem $(\dot{\bar{c}} \leq 0$ towards the IR) and writing $\dot{\bar{c}} \sim \dot{\lambda}^{i} \partial \bar{c} / \partial \lambda^{i}$, it is easy to derive the modification of the $c$-theorem by gravity at the vicinity of fixed points with central charge $c$ :

$$
\ddot{\bar{c}}+Q \dot{\bar{c}}\left\{\begin{array}{l}
\leq 0 \text { for } c>25 \\
\geq 0 \quad \text { for } c \leq 1,
\end{array} \quad \text { with } \quad Q^{2}=\frac{1}{3}|c-25| .\right.
$$

Here, the dot represents derivative with respect to $t$, not $\tau$.

A second qualitative effect of gravity concerns flows that start from $c>25$ but do not reach a fixed point with $c \geq 25$. They obey eq.(2.14), which is valid everywhere (i.e., not only near fixed points) up to order $\alpha^{\prime}$ :

$$
\dot{Q}=\frac{c(\vec{\lambda})-25}{3}-Q^{2} .
$$

Note that if $Q$ is real, it stays real at all times. Since $Q^{2}$ would have to be negative at fixed points with $c<25, \vec{\lambda}(t)$ cannot converge to a CFT with $c<25$. It is possible for $\bar{c}(\vec{\lambda})$ to become smaller than 25 , but then $\dot{\vec{\lambda}}$ must be large enough to make $Q^{2} \geq 0$ in (2.22). We conclude that in the models with dynamical $2 d$ gravity there is a ' $c=25$ barrier' for the flow: a flow that starts with $c>25$ is either attracted to a fixed point with $c \geq 25$, or it does not converge to a fixed point at all (see also the example in the next section). This result, derived here to the leading order in $\alpha^{\prime}$, may be true to all orders.

\section{An example: Group space}

The purpose of this section is to illustrate some of the preceding discussion on a 'minisuperspace' example, considered previously in [2], with solutions which interpolate between a flat space and a group space (the Wess-Zumino-Witten model). Consider the sigma model with a WZW term 40]. The $N$-dimensional target space is the group space $G$ with a fixed metric $\hat{G}_{i j}$ and curvature

$$
\hat{R}_{i j}^{(N)}=\frac{1}{4} f_{i m n} f_{j}^{m n}=\frac{1}{4} c_{G} \hat{G}_{i j}
$$

\footnotetext{
${ }^{19}$ Note that the $c$-theorem in its original formulation [3] does not apply to sigma models and in the presence of (non-unitary) dilaton (or ghost) interaction terms, see [28, 25, 29, 41].
} 
where $f_{i j k}$ are the structure constants and $c_{G}$ is the value of the quadratic Casimir operator in the adjoint representation. The WZW term can be represented by the antisymmetric tensor field $\hat{B}_{i j}$ with the field strength

$$
\hat{H}_{i j k}=k f_{i j k} \text {. }
$$

For the time dependence of the background we make the ansatz

$$
\begin{aligned}
G_{i j}(\vec{x}, t) & =\frac{1}{g^{2}(t)} \hat{G}_{i j}=e^{2 \lambda(t)} \hat{G}_{i j}(\vec{x}) \\
\phi(\vec{x}, t) & =\phi(t) \\
H_{i j k} & =\hat{H}_{i j k}=k f_{i j k} .
\end{aligned}
$$

This ansatz is consistent as a consequence of the group symmetry; one can also show that, as expected, there are no solutions with time-dependent $k$. $\lambda$ and $\phi$, or $\lambda$ and $\varphi=2 \phi-N \lambda$ are the coupling constants of the model. For the minkowskian sector of solutions with $G^{00}=-1$ the equations for $\lambda(t)$ and $Q \equiv-\dot{\varphi}(t)$ are (to order $\alpha^{\prime}$ ) 11]

$$
\begin{aligned}
\ddot{\lambda}+Q \dot{\lambda} & =-\frac{1}{6 N} \frac{\partial \bar{c}(\lambda)}{\partial \lambda}, \\
Q^{2} & =N \dot{\lambda}^{2}+\frac{1}{3}[\bar{c}(\lambda)-25] \\
\dot{Q} & =-N \dot{\lambda}^{2} .
\end{aligned}
$$

Here the $\bar{c}$-function (2.15),(2.4) is

$$
\bar{c}(\lambda)=C^{(N)}=N-3\left(R^{(N)}-\frac{1}{12} H^{2}\right)=N+\frac{3}{4} N c_{G}\left(-e^{-2 \lambda}+\frac{1}{3} k^{2} e^{-6 \lambda}\right) .
$$

For comparison, the standard RG flow towards the IR region in the $N$-dimensional model is determined by

$$
\dot{\lambda}=\beta(\lambda)=-\frac{1}{6 N} \frac{\partial \bar{c}(\lambda)}{\partial \lambda}=-\frac{1}{4} c_{G} e^{-2 \lambda}\left(1-k^{2} e^{-4 \lambda}\right) .
$$

Near fixed points (5.4)-(5.6) agree with (2.20), (2.22), (2.23) (up to a normalisation of $\lambda ; \dot{\lambda}^{2}$ terms are absent in this case). $\bar{c}(\lambda)$ approaches $N$ from below for $\lambda \rightarrow \infty(\lambda=\infty, \bar{c}=N$ is a trivial fixed point) and has the minimum (corresponding to the well-known zero of $\beta(\lambda)$ 40]) at

$$
e^{2 \lambda}=|k|, \quad \bar{c}_{\text {min }}=N-\frac{c_{G} N}{2|k|}+O\left(\frac{1}{k^{2}}\right)=\frac{N|k|}{|k|+\frac{1}{2} c_{G}} .
$$

We are restricted to large enough $\lambda$ so that higher order corrections in $\alpha^{\prime}$ can be neglected.

The above equations illustrate the following points of sec. 3 (points 1,2 and 4) and of sec. 4 (point 3):

1. Eqs (5.4)-(5.6) with $Q>0$ describe a damped motion of a particle in the potential $\bar{c}(\lambda)$. The model can be viewed as interpolating between different internal spaces, namely, a flat space (the asymptotically free limit $\lambda \rightarrow \infty$ of the sigma model) and a curved 
space (the WZW model). If $\bar{c}_{\text {min }}>25, \lambda$ settles down - after possible oscillations - at the WZW fixed point ('compactification'), while for $\bar{c}_{\text {min }}<N<25$ (corresponding to the Euclidean sector of solutions) the model approaches (at large Euclidean time) the flat space ('decompactification' in Euclidean time).

2. If $c_{\text {min }}=25$, the solutions are still attracted to the WZW model. In its vicinity (where the potential $\bar{c}$ is proportional to $\lambda^{2}$ where $\lambda$ has been shifted such that it is zero at the WZW point), $\vec{\lambda}(t)$ performs oscillations with an amplitude $\Lambda(t)$ and the energy $Q^{2}(t)$ which satisfies

$$
\begin{array}{r}
Q^{2}=E_{k i n}+E_{p o t}, \quad \frac{d}{d t} Q^{2}=-2 N Q \dot{\lambda}^{2} \\
E_{k i n}=N \dot{\lambda}^{2}, \quad E_{\text {pot }}=\frac{1}{3}[\bar{c}(\lambda)-25] .
\end{array}
$$

Due to the damping, $\Lambda$ and $Q$ can be seen to slowly decrease with time : $Q, \Lambda \sim t^{-1}$.

3. If $N>25$ but $\bar{c}_{\text {min }}<25$, there is no stable fixed point. Indeed, starting from the flat space model with $\bar{c}=N$, eq.(5.6) implies that $Q$ decreases until a new fixed point is reached. But the WZW fixed point with $\bar{c}_{\min }<25$ cannot be reached since it would correspond to imaginary $Q$, and there is no other fixed point with $c \geq 25$. So $Q$ keeps decreasing, becomes negative, and the solution blows up due to anti-damping ${ }^{20}$ (of course, at some point terms of higher orders in $\alpha^{\prime}$ must be taken into account). Applied to the RG flow in the presence of $2 d$ gravity, this illustrates that starting from a fixed point with $c>25$ the flow cannot reach another fixed point with $c<25$ even though this was possible in the absence of coupling to gravity. Instead, the flow diverges.

4. Near the WZW fixed point, the $\lambda$-perturbation has dimension $>2$, so for $c_{\min }>25$ the WZW point is an attractor within this 'minisuperspace'. The other fixed point, corresponding to the free model, is unstable although it has no operators with dimension less than two (the $\lambda$-perturbation has dimension two at the free end). The reason is a negative quadratic $\beta$-function or OPE coefficient. The requirement of not having negative quadratic beta functions for operators with dimension two is thus a nontrivial restriction on attractors.

\section{Concluding remarks}

Viewing cosmological solutions of string theory as trajectories in the space of $2 d$ field theories, we have seen that while the trajectories diverge for some domains of initial values, there are domains within which the trajectories are attracted to the minima of $|\bar{c}-25|$. In particular, minima with $\bar{c}=25$ are also attractors. As mentioned in the introduction, one hope is to assign, already at the classical level, different probabilities to different string vacua, proportional to the sizes of the corresponding domains of attraction.

\footnotetext{
${ }^{20} \mathrm{~A}$ similar remark applies to solutions that start from the WZW fixed point with increasing $\bar{c}$.
} 
The present discussion cannot explain why the universe is not stuck, e.g., in a minimum of $\bar{c}$ with $\bar{c}>25$. But our analysis was restricted to classical string theory. One may speculate that in the quantum theory the universe can tunnel between various minima of $|\bar{c}-25|$ until it reaches a ground state with $\bar{c}=25$.

Quantum effects in string theory are described by sums over world sheets of different topologies. In view of the conjectured interpretation of (some of the) cosmological string solutions as renormalisation group trajectories in the presence of dynamical $2 d$ gravity, one may wonder whether the only IR-stable fixed points of two-dimensional field theory on a surface with fluctuating geometry and topology are critical $(c=25)$ string vacua.

\section{Acknowledgements:}

The work of C.S. is supported by Schweizerischer Nationalfonds. A.A.T. would like to acknowledge the support of PPARC.

\section{References}

[1] R.C. Myers, Phys. Lett. B199 (1988) 371.

[2] I. Antoniadis, C. Bachas, J. Ellis and D.V. Nanopoulos, Nucl. Phys. B328 (1989) 117; Phys. Lett. B211 (1988) 393.

[3] A.B. Zamolodchikov, JETP Lett. 43 (1986) 731; Sov. J. Nucl. Phys. 46 (1987) 1090.

[4] M. Mueller, Nucl. Phys. B337 (1990) 37; G. Veneziano, Phys. Lett. B265 (1991) 287; A.A. Tseytlin and C. Vafa, Nucl. Phys. B372 (1992) 443.

[5] A. Sen, Phys. Lett. B252 (1990) 566.

[6] C. Lovelace, Phys. Lett. B135 (1984) 75; Nucl. Phys. B273 (1986) 413.

[7] E.S. Fradkin and A.A. Tseytlin, Phys. Lett. B158 (1985) 316; Nucl. Phys. B261 (1985) 1.

[8] C.G. Callan, D. Friedan, E.J. Martinec and M.J. Perry, Nucl. Phys. B262 (1985) 593.

[9] T. Banks and E. Martinec, Nucl. Phys. B294 (1987) 733; J. Hughes, J. Liu and J. Polchinski, Nucl. Phys. B316 (1989) 15; J. Schnittger and U. Ellwanger, preprint HDTHEP-92-48, hep-th/9211139.

[10] A. Cooper, L. Susskind and L. Thorlacius, Nucl. Phys. B363 (1991) 132; in: Strings and Symmetries, Proc. of 1991 Stony Brook conference (World Scientific, Singapore 1992), p. 225.

[11] A.A. Tseytlin, Int. J. Mod. Phys. D1 (1992) 223.

[12] S. Mukherji, Mod. Phys. Lett. A7 (1992) 1361.

[13] A.M. Polyakov, Mod. Phys. Lett. A2 (1987) 893; V.G. Knizhnik, A.M. Polyakov and A.B. Zamolodchikov, Mod. Phys. Lett. A3 (1988) 819. 
[14] J. Ellis, N.E. Mavromatos and D.V. Nanopoulos, Phys. Lett. B293 (1992) 37; CERN preprints TH.6755, TH.6896, TH.6897, TH.7000; in Proceedings of the HARC Workshop on recent advances in the superworld, Woodlands, April 14-16, 1993.

[15] S. Das, S. Naik and S. Wadia, Mod. Phys. Lett. A4 (1990) 1033; S. Das, A. Dhar and S. Wadia, Mod. Phys. Lett. A5 (1990) 799.

[16] T. Banks and J. Lykken, Nucl. Phys. B331 (1990) 173; T. Banks, in: Physicalia Magazine, vol.12 (Gent 1990).

[17] A.A. Tseytlin, Int. J. Mod. Phys. A5 (1990) 1833.

[18] A.M. Polyakov, Lectures in Les Houches, July 1992; hep-th/9304146.

[19] C. Schmidhuber, Nucl. Phys. B404 (1993) 342.

[20] I. R. Klebanov, I. I. Kogan and A. M. Polyakov, Phys. Rev. Lett. 71 (1993) 3243.

[21] J. Ambjorn and K. Ghoroku, NBI preprint NBI-HE-93-63 (1993).

[22] Y. Tanii, S. Kojma and N. Sakai, Phys. Lett. B322 (1994) 59.

[23] F. David, Mod. Phys. Lett. A3 (1988) 1651.

[24] J. Distler and H. Kawai, Nucl. Phys. B321 (1988) 509.

[25] G. Curci and G. Pafutti, Nucl. Phys. B286 (1987) 399; Nucl. Phys. B312 (1989) 227.

[26] A.A. Tseytlin, Int. J. Mod. Phys. A5 (1990) 1833.

[27] K.A. Meissner and G. Veneziano, Phys. Lett. B267 (1991) 33.

[28] A.A. Tseytlin, Phys. Lett. B194 (1987) 63; Phys. Lett. B223 (1989) 165.

[29] H. Osborn, Phys. Lett. B214 (1988) 555; Nucl. Phys. B308 (1988) 629; S. De Alwis, Phys. Lett. B217 (1989)467.

[30] A.A. Tseytlin, Phys. Lett. 178 (1986) 34; Nucl. Phys. B294 (1987) 383.

[31] C.M. Hull and P.K. Townsend, Nucl. Phys. B274 (1986) 349; G.M. Shore, Nucl. Phys. B286 (1987) 349; H. Osborn, Nucl. Phys. B363 (1991) 486; Ann. of Phys. 200 (1990) 1.

[32] J.H. Cardy, Les Houches summer school, session XLIX, 1988 (North Holland, Amsterdam 1989).

[33] J. Polchinski, Nucl. Phys. B324 (1989) 123.

[34] G. Moore, Yale preprint YCTP-P1-92, hep-th/9203061.

[35] N. Seiberg, Prog. Theor. Phys. S102 (1990) 319;

[36] J. Polchinski, Nucl. Phys. B362 (1991) 125.

[37] A. Anderson, B.E.W. Nilsson, C.N. Pope and K.S. Stelle, preprint CTP TAMU-1/94, hep-th/9401007.

[38] U.H. Danielsson, CERN preprint CERN-TH.7155/94 (1994), hep-th/9401135.

[39] J. Polchinski, Nucl. Phys. B346 (1990) 253. 
[40] E. Witten, Commun. Math. Phys. 92 (1984) 455.

[41] S. Elitzur, A. Giveon and E. Rabinovici, Nucl. Phys. B316 (1989) 679; P. Mende, Phys. Rev. Lett. 63 (1989) 89. 